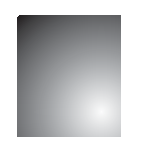

\title{
Produção Científica em Finanças: mapeamento das PUblicações em Periódicos Qualis A no Brasil
}

\author{
Scientific Production in Finance: mapping of publications in \\ journals Qualis A in Brazil
}

\section{Luiz Henrique Debei Herling}

Mestre em Finanças. Universidade Federal de Santa Catarina. Florianópolis, SC. Brasil. E-mail: lhherling@gmail.com.

Gilberto de Oliveira Moritz

Professor do Departamento de Ciências da Administração da Universidade Federal de Santa Catarina. Florianópolis, SC. Brasil.

E-mail: gomoritz@cse.ufsc.br

Thiago Coelho Soares

Mestre em Administração. Universidade Federal de Santa Catarina. Florianópolis, SC. Brasil. E-mail: tcoelhos@hotmail.com

Marcus Venícius Andrade de Lima

Doutor em Engenharia de Produção. Universidade Federal de Santa Catarina. Florianópolis, SC. Brasil. E-mail: marcus.lima@ufsc.br

\section{Resumo}

O atual campo da administração financeira está ligado diretamente à Ciência da Administração, herdando traços de seu anterior berço: as Ciências Econômicas. Muitos são os estudos publicados em finanças ao longo dos anos, porém, o que a administração financeira estuda não é algo de simples evidenciação. Há 70 anos, pesquisadores da área questionam e tentam fazer uma aproximação do que esse campo de atuação trata. Por meio de um estudo descritivo, buscou-se entender como estão as publicações na área de administração financeira nas revistas classificadas de alto impacto, de acordo com o último Qualis publicado pela Capes no ano de 2012. Através da pesquisa verificou-se que, entre 2010 até 2012, existe uma concentração maior de pesquisas nas subáreas Finanças de Curto Prazo, Finanças de Longo Prazo e Mercados e Instituições Financeiras; que os pesquisadores com maior produtividade são da Universidade Presbiteriana Mackenzie e da Fundação Getúlio Vargas (EAESP/FGV); e que as revistas RAUSP e RAE apresentam a maior quantidade de artigos publicados sobre finanças.

Palavras-chave: Administração Financeira. Pesquisas em Finanças. Publicações em Finanças.

\section{Abstract}

The current field of financial management is directly linked to the science of Directors also inheriting traits of his crib before then, the economic sciences. There are many published studies in finance over the years, but the very definition of what financial management is studying is not something simple disclosure. About seventy years ago, researchers in the field have questioned and tried to make an approximation of what this field of operation aim to treat. Through a descriptive study as quantitative treatment of the data using descriptive statistics, we sought to understand how the publications are in the area of financial management in high-impact journals classified according to the last published qualis Capes in 2012. Through research showed that between 2010 until 2012 there is a greater concentration of research in Finance Short Term Finance Long Term Markets and Financial Institutions subareas, researchers with higher productivity are of Mackenzie University and Getúlio Vargas Foundation (EAESP/FGV), and that RAUSP and RAE magazines have the largest amount of published articles on finance.

Keywords: Financial Management. Research in Finance. Finance Publications. 


\section{INTRODUÇÃO}

Solomon (1977) afirma que não existe uma definição do âmbito da Administração Financeira para estudo acadêmico, que não existe uma resposta definitiva, mas que existe uma grande quantidade de possíveis aproximações.

Ao passar pelos clássicos internacionalmente reconhecidos pelas suas publicações em finanças, desde os tempos em que esses estudos estavam inerentes à Ciência Economia, passando por Galbraith (1977) e Keynes (1985), até ser incorporada no escopo da Ciência da Administração aprofundadas por Weston (1977), Salomon (1977), Gitman (2010), Ross (1995), Brealey (2006), tem-se a administração financeira definida de maneira unânime e sintetizada pelos conceitos desses autores como a arte de administrar os recursos de uma organização.

Para Assaf Neto (2005), um dos objetivos da administração financeira é maximizar o valor de mercado do capital investido dos proprietários, sendo assim esse objetivo deve estar em sintonia com o que o acionista almeja, ou seja, a remuneração do seu capital, sob a forma de dividendos e/ou lucros.

A gestão financeira pode ser dividida ainda em gestão de longo prazo e gestão de curto prazo. Segundo Matias (2007), as finanças podem ser divididas em macro e micro finanças. No que tange às macrofinanças, é preciso considerar aspectos relacionados à economia $e$ às questões de fluxos internacionais. As microfinanças são aquelas voltadas de certa forma ao ambiente interno da empresa. Esse ambiente interno é dividido ainda em gestão de curto prazo e gestão de longo prazo. Em uma definição superficial, pode-se dizer que a gestão de longo prazo contempla temas como análise de projetos, estrutura e orçamento de capital. No curto prazo, contempla a gestão do capital de giro.

Essa classificação, no entanto, já vem de discussões mais antigas, em que Weston (1975) trata dos problemas da definição da função financeira. Anteriormente, ainda, tem-se os estudos de Pearson Hunt (1940), que propõe não existir consonância entre a prática acadêmica e o mundo profissional. Sabe-se que a academia vem fornecendo por meio de suas pesquisas subsídio ao mundo corporativo $e$ econômico para a tomada de decisões em diversos campos de atuação.
No Brasil, ainda há as particularidades da nossa economia $e$ as diferentes abordagens que em muitas oportunidades se distinguem da literatura que é predominantemente desenvolvida sobre o enfoque industrial norte-americano. Alguns autores, como Assaf Neto (2005), Lemes Jr, Cherobim (2002) e Matias (2006), fazem adaptações ao contexto brasileiro a fim de repensar os modelos propostos em nosso contexto.

No âmbito dos periódicos científicos, diversos são os estudos publicados na área de finanças dentre os 1.503 periódicos classificados pelo Qualis da Capes, sendo classificadas como A1 e A2 as publicações de alto impacto. Limitando para as publicações de alto impacto no Brasil, há 29 periódicos em que as publicações são realizadas. É de grande importância o conhecimento dessas publicações, para saber como a academia vem produzindo estudos e soluções frente aos desafios do mundo contemporâneo. Diante disso, o presente estudo busca evidenciar como estão as publicações em periódicos Qualis A no Brasil.

\section{Fundamentação TeÓRICA}

Para compreender melhor a relação entre evolução da função financeira e acontecimentos históricos, Weston (1977) partiu de três proposições básicas. A primeira é que a história do pensamento econômico gera reflexos em problemas prementes existentes em cada momento histórico. A segunda é que novos avanços em Finanças estão relacionados com o desenvolvimento, em campos correlacionados, de teorias einstrumentos. A terceira proposição é que, em Finanças, as transformações sofridas em campos relacionados possibilitaram que se desse pouca atenção a certos aspectos de seu conteúdo em uma época e que fosse dada a devida consideração em outras.

A crescente complexidade assumida pelos negócios e operações de mercado fez com que as finanças empresariais se separassem das Ciências Econômicas. Até 1929/1930 existia o predomínio da abordagem tradicional, em que as finanças estão voltadas para instrumentos de captação de recursos. Com a depressão de 1929, o enfoque administrativo passa a existir e a preocupação com solvência e liquidez das empresas. Na década de 1940 até meados de 1950, o enfoque passa a ser da empresa como aplicadora (empréstimo) 
de recursos. Na década de 1950, a ênfase é dada ao investimento, à geração de riqueza e à preocupação com alocação eficiente de recursos. Os conceitos de Retorno do investimento e custo de capital foram desenvolvidos. No final de 1950 e início de 1960, Modigliani e Miller deram início à moderna teoria de finanças com estudos sobre a irrelevância da estrutura de capital. Na década de 1990, a evolução da gestão de risco, com estratégias de derivativos, opções, swaps, hedges, etc.

O objetivo de uma empresa é a maximização dos lucros.

A razão lógica fundamental do objetivo da maximização da riqueza, é que essa maximização reflete a utilização mais eficiente dos recursos econômicos de uma sociedade, promovendo, assim, a maximização da riqueza econômica da sociedade. (SOLOMON, 1977, p. 40)

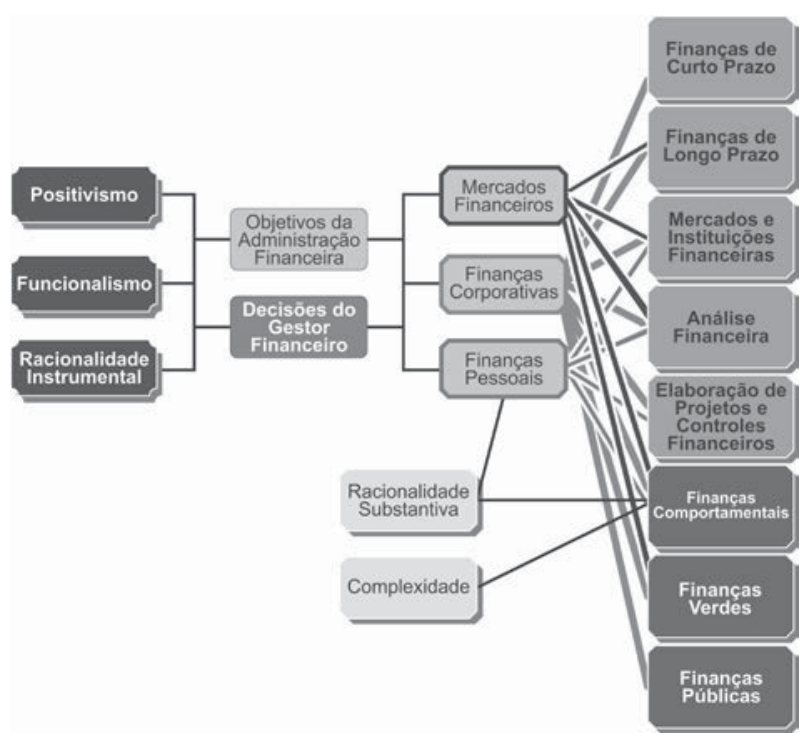

Figura 1: Epistemologia de finanças

Fonte: Herling, Moritz e Lima (2013)

As funções da administração financeira podem ser apresentadas em quatro grupos: planejamento $e$ controle financeiro; decisões de investimento e decisões de financiamento. Planejamento Financeiro: tem como objetivo definir com antecedência os fatos ou ações futuras dentro de cenários preestabelecidos, visando a maximização dos resultados, delegando ao responsável de cada área na organização, o cumprimento dos objetivos e metas predeterminadas. Atividades de Investimento: são classificadas as atividades que refle- tem as decisões de aplicações de recursos temporários ou permanentes, por meio da seleção dos ativos mais rentáveis e condizentes com os negócios da empresa. Atividades de Financiamento: são classificadas as atividades que refletem as decisões tomadas para definir o tipo, forma e prazo dos financiamentos captados, seja para a operação ou para investimentos. Volta-se para a aquisição de fundos para o gerenciamento de sua composição, procurando definir a estrutura mais adequada em termos de liquidez, redução de seus custos financeiros e risco financeiro. (ASSAF NETO, 2005)

Finanças Corporativas - estão relacionadas à decisão sobre a estrutura de capital, ao controle e à propriedade das empresas, ao processo de emissão de títulos e à governança corporativa. (LEAL; SAITO, 2003)

Mercados Financeiros - mercado voltado para a transferência de recursos entre os poupadores e os investidores. No mercado financeiro são efetuadas transações com títulos de prazos médios, longos e indeterminados, geralmente dirigidas ao financiamento dos capitais de giro e fixo. (ASSAF NETO, 2005)

Finanças Pessoais - têm por objeto de estudo e análise as condições de financiamento das aquisições de bens e serviços necessários à satisfação das necessidades e desejos individuais. (PIRES, 2007)

Finanças de Longo Prazo - envolvem recursos financeiros com prazo de recebimento ou pagamento superior a um ano. Estão relacionadas ao passivo e ao ativo não circulante do balanço patrimonial. (ROSS, 1995)

Mercados e Instituições Financeiras - são os mercados e as instituições, relacionados ao mercado financeiro, que têm a função de intermediar os agentes superavitários - aqueles que têm excesso de recursos e os aplicam no mercado financeiro; e os agentes deficitários aqueles que têm escassez de recursos em dado momento e precisam recorrer a fontes para equilibrar sua situação de caixa. (ROBERTS, 2000)

Finanças de Curto Prazo - envolvem recursos financeiros que envolvam prazo de recebimento ou pagamento inferior a um ano. Está relacionado ao passivo e ativo circulante do balanço patrimonial. (ROSS, 1995)

Finanças Comportamentais - ramo do estudo de finanças que tem como objetivo a revisão e o aperfeiçoamento do modelo econômico-financeiro atual, pela incorporação de evidências sobre a irracionalidade do investidor. (HALFELD; TORRES, 2001) 
Análise Financeira - metodologia de análise que se baseia na análise dos demonstrativos financeiros (balanço patrimonial, demonstrativo de resultado e demonstração de origens e recursos) de uma empresa para determinar a sua posição financeira atual assim como desempenho futuro. A análise financeira é útil porque em geral o desempenho passado de uma empresa é um bom indicador de seu desempenho futuro, assim como a situação financeira atual é o suporte para o crescimento futuro. (ASSAF NETO, 2005)

Finanças Públicas - conjunto de recursos e de regras que concernem à atividade financeira das administrações públicas. (SANTOS; ALVES, 2011)

Elaboração de Projetos e Controle Financeiro - a execução do processo de controle financeiro consiste na verificação do cumprimento das metas e dos objetivos previamente estabelecidos. (ROCHA et al., 2011)

Finanças Ambientais - aborda os temas ambientais, sociais e econômicos. (FRANCO, 2009)

\section{Metodologia}

O principal interesse deste estudo é evidenciar como estão as publicações em periódicos Qualis A no Brasil. A técnica utilizada foi uma meta-análise dos artigos empíricos de periódicos constantes na revisão da literatura.

A meta-análise é uma revisão bibliográfica sistemática. $\mathrm{Na}$ área das ciências sociais, justifica-se pela necessidade de utilização de métodos que possibilitem trazer à tona as contradições, transformando-as em conhecimento (BOTELHO et al., 2011). A meta-análise visa extrair informação adicional de dados preexistentes através da união de resultados de diversos trabalhos e pela aplicação de uma ou mais técnicas estatísticas. Trata-se de um método quantitativo que permite combinar os resultados de estudos já realizados (extraídos de trabalhos publicados) e sintetizar as suas conclusões ou mesmo extrair uma nova conclusão. (LUIZ, 2002)

Para se configurar como uma meta-análise não basta que se analisem qualitativamente os resultados de trabalhos anteriores, como em uma revisão. É imprescindível uma nova análise estatística dos dados, ou resultados reunidos, para que o processo receba essa designação. (LUIZ, 2002)

\subsection{Pesquisa}

O estudo tem sua natureza metodológica em uma abordagem descritiva ao dispor como as publicações em finanças se encontram nos periódicos classificados como A1 e A2 nacionais, seu enquadramento quanto à área de estudos em administração financeira, conforme a Epistemologia de Finanças proposta por Herling, Moritz e Lima (2013). Os periódicos foram selecionados de acordo com o último relatório expedido pela Capes, e recortados apenas os periódicos nacionais que estejam classificados como A1 e A2. Foram selecionados 29 periódicos, em que foram todos acessados e buscados artigos ali publicados, sem corte de caráter temporal, publicações que contenham em resumo, texto e título um dos seguintes termos: Finanças, Financeiro, Financeira e no inglês Financial.

Posteriormente os artigos foram classificados nesta ordem: título, revista, área em finanças e ano de publicação. Para tanto, utilizou-se uma abordagem quantitativa através do tratamento dos dados por meio de estatística descritiva.

\section{Apresentação dos Resultados}

Nesta seção serão apresentadas a descrição $e$ a análise dos dados, demonstrando os resultados do estudo bibliométrico e do mapeamento da pesquisa. Após o levantamento dos artigos, os resultados serão apresentados a seguir.

A primeira classificação desenvolvida foi com base nas decisões financeiras abordadas no referencial teórico. Essa classificação poderia ser com base em: decisões de planejamento e controle financeiro, decisões de investimento e decisões de financiamento.

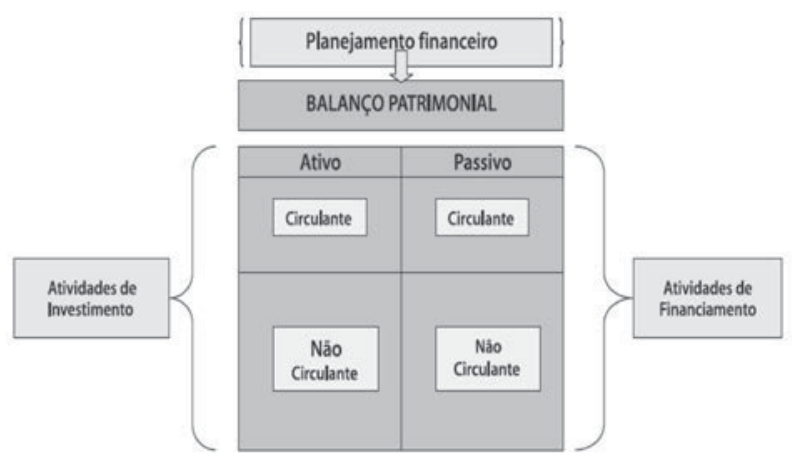

Figura 2: Classificação das finanças

Fonte: Adaptada de Assaf Neto (2005) 
Os resultados para essa classificação inicial estão expressos no Gráfico 1, a seguir.

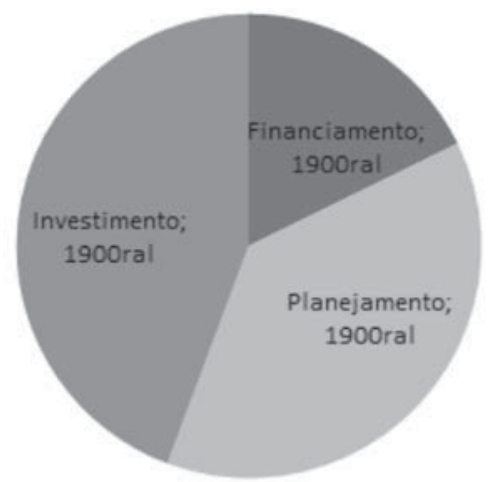

Gráfico 1: Resultados da classificação das finanças Fonte: Elaborado pelos autores deste artigo

Observa-se uma concentração maior nas decisões de investimento e planejamento em detrimento a pesquisas em decisões de financiamento. As decisões de investimento totalizam 35 artigos encontrados. Essa maior quantidade se deve a pesquisas feitas na área de mercados financeiros.

A segunda classificação desenvolvida foi com base nas áreas de finanças abordadas no referencial teórico. Essa classificação poderia ser baseada em: mercados financeiros, finanças corporativas e finanças pessoais, conforme a figura a seguir.

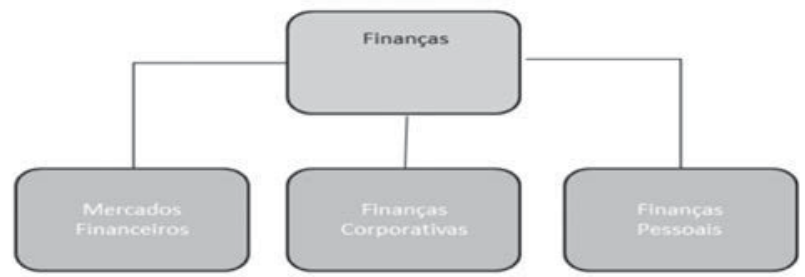

Figura 3: Segunda classificação de finanças

Fonte: Elaborada pelos autores deste artigo

Assim, foram classificadas as áreas mais publicáveis conforme a figura a seguir.

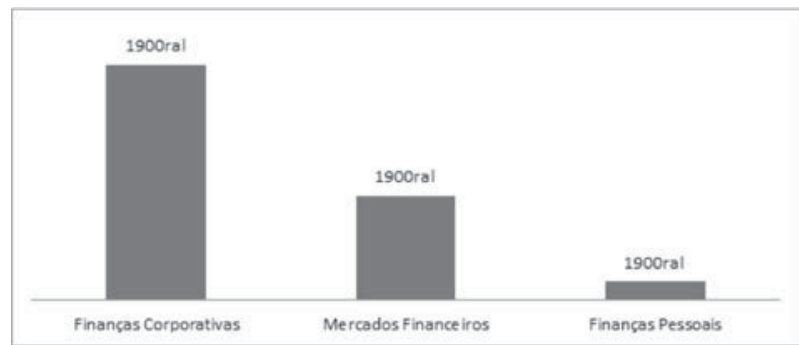

Gráfico 2: Resultados da segunda classificação das finanças Fonte: Elaborado pelos autores deste artigo
Os resultados encontrados estão relacionados, em sua maior parte, a duas áreas: finanças corporativas e mercados financeiros. Essas duas áreas concentram a maioria das pesquisas desenvolvidas nos periódicos qualis A.

Para a classificação nas subáreas utiliza-se os esquemas a seguir. Nele tem-se as subáreas tradicionais em finanças (vertical) e as novas (horizontal).

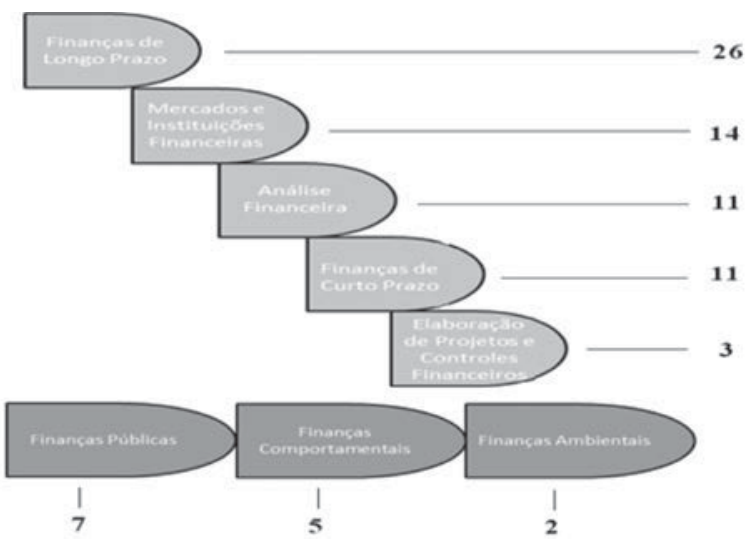

Figura 4: Classificação das subáreas de finanças Fonte: Elaborado pelos autores deste artigo

Aplicando essa classificação aos artigos estudados, é possível encontrar os resultados a seguir.

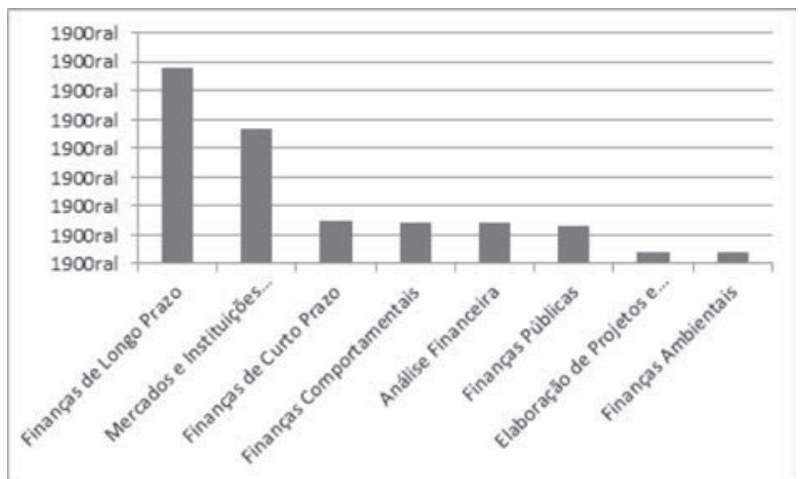

Gráfico 3: Resultados da classificação das subáreas de finanças Fonte: Elaborado pelos autores deste artigo

As pesquisas entre as subáreas estão concentradas em sua maior parte em finanças de longo prazo e mercados e instituições financeiras. Essas duas áreas são as únicas que apresentam mais de 15 artigos publicados ao longo desses anos em periódicos Qualis A.

Dentre as novas subáreas de finanças, é possível destacar a produção com temas referentes a finanças comportamentais e a finanças públicas.

A seguir será apresentada a evolução do volume de publicação por ano. 


\begin{tabular}{|c|c|}
\hline Ano & Percentual das Publicações \\
\hline 1967 & $1,27 \%$ \\
\hline 1974 & $1,27 \%$ \\
\hline 1989 & $1,27 \%$ \\
\hline 1992 & $1,27 \%$ \\
\hline 1995 & $1,27 \%$ \\
\hline 1996 & $1,27 \%$ \\
\hline 1997 & $3,80 \%$ \\
\hline 1999 & $1,27 \%$ \\
\hline 2000 & $2,53 \%$ \\
\hline 2001 & $2,53 \%$ \\
\hline 2002 & $3,80 \%$ \\
\hline 2003 & $5,06 \%$ \\
\hline 2005 & $6,33 \%$ \\
\hline 2006 & $2,53 \%$ \\
\hline 2007 & $12,66 \%$ \\
\hline 2008 & $12,66 \%$ \\
\hline 2009 & $8,86 \%$ \\
\hline 2010 & $5,06 \%$ \\
\hline 2011 & $11,39 \%$ \\
\hline 2012 & $12,66 \%$ \\
\hline
\end{tabular}

Quadro 1: Resultados da evolução de pesquisas por ano Fonte: Elaborado pelos autores deste artigo

O Gráfico 4 resume o número de publicações por ano sobre finanças de forma cumulativa. Estes dados devem ser interpretados com cautela por dois motivos. Em primeiro lugar, as revistas da Scielo contêm mais informações sobre suas publicações mais recentes. Em segundo lugar, não há dúvida que existe uma tendência crescente na comunidade acadêmica para publicar. Com essas advertências em mente, no entanto, é evidente que o interesse não está diminuindo no assunto.

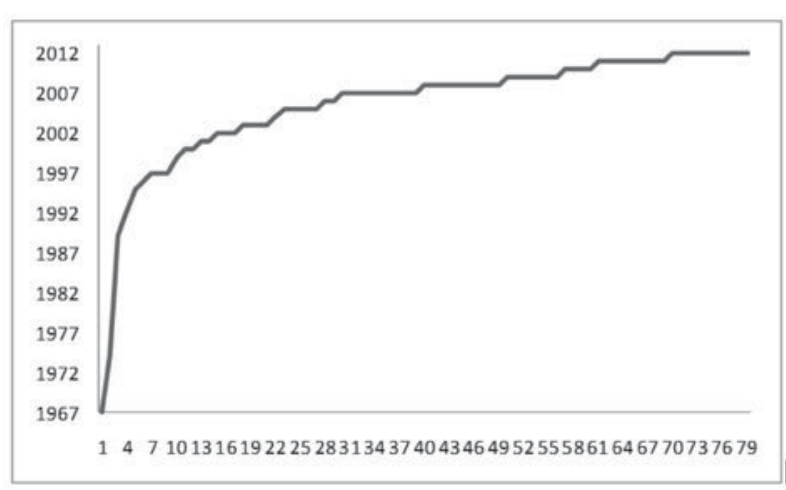

Gráfico 4: Resultados da publicação por ano de forma cumulativa

Fonte: Elaborado pelos autores deste artigo

Considerando as revistas mais prolíficas, tem-se o quadro a seguir.

\begin{tabular}{|c|c|}
\hline NOME DE PERIÓdico & $\begin{array}{c}\text { NúMERo } \\
\text { DE ARTIGos } \\
\text { PUBLICADOS }\end{array}$ \\
\hline RAE & 23 \\
\hline BAR Brazilian Administration Review & 10 \\
\hline Revista de Administração Contemporânea & 8 \\
\hline RAC Eletrônica & 6 \\
\hline Ciência e Saúde Coletiva (Impresso) & 5 \\
\hline RAE Eletrônica & 5 \\
\hline Cadernos de Saúde Pública (ENSP. Impresso) & 4 \\
\hline BBR Brazilian Business Review & 3 \\
\hline Organizações \& Sociedade (Impresso) & 3 \\
\hline Ciência e Agrotecnologia (UFLA) & 2 \\
\hline Gestão \& Produção (UFSCAR) & 2 \\
\hline Revista Brasileira de Economia & 2 \\
\hline Revista Contabilidade \& Finanças & 2 \\
\hline Engenharia Agrícola & 1 \\
\hline Estudos Avançados (USP) & 1 \\
\hline RAP & 1 \\
\hline Revista Árvore & 1 \\
\hline
\end{tabular}

Quadro 2: Resultados das revistas que mais publicaram artigos em finanças

Fonte: Elaborado pelos autores deste artigo

De acordo com o Quadro 2, é possível observar que as revistas RAUSP e RAE se destacam. A RAUSP 
é a Revista de Administração da USP que apresentou a maior quantidade de artigos publicados sobre finanças, sendo 46 no total. A RAE, segunda revista mais prolífica, teve metade da quantidade publicada pela RAUSP.
Ao longo da pesquisa foram identificados 171 autores (o quadro completo se encontra no anexo). Considerando as revistas mais prolíficas, ou seja, as que apresentaram pelo menos um artigo nas revistas pesquisadas, tem-se o quadro a seguir.

\begin{tabular}{|c|c|c|}
\hline \multicolumn{1}{|c|}{ Autor } & INSTITução & NúMERo ARTIGOS \\
\hline Wesley Mendes-da-Silva & Universidade Presbiteriana Mackenzie & 4 \\
\hline Herbert Kimura & Universidade Presbiteriana Mackenzie & 3 \\
\hline Luiz Artur Ledur Brito & Fundação Getúlio Vargas - EAESP/FGV & 3 \\
\hline Wilson Toshiro Nakamura & Universidade Presbiteriana Mackenzie & 3 \\
\hline Adalberto Schnorrenberger & Univates & 2 \\
\hline Jairo Laser Procianoy & Universidade Federal do Rio Grande do Sul, & 2 \\
\hline John Robison & Unisersityof Texas & 2 \\
\hline Raimundo Christians & Prince Waterhouse & 2 \\
\hline Ricardo Pereira Câmara Leal & UFRJ Universidade Federal do Rio de Janeiro & 2 \\
\hline Saul B. Suslick & Universidade Estadual de Campinas/UNICAMP & 2 \\
\hline Stephen Limberg & Unisersityof Texas & 2 \\
\hline
\end{tabular}

Quadro 3: Resultados dos autores que mais publicaram artigos em finanças

Fonte: Elaborado pelos autores deste artigo

Observa-se que os pesquisadores com maior produtividade são Wesley Mendes-da-Silva, Herbert Kimura, Luiz Artur Ledur Brito e Wilson Toshiro Nakamura. Destes, três são da Universidade Presbiteriana Mackenzie e um da Fundação Getúlio Vargas - EAESP/FGV.

Com a discriminação dos autores em gênero, verifica-se que os autores do sexo masculino publicaram 145 artigos, enquanto os autores do sexo feminino publicaram 42 artigos.

O gráfico a seguir evidencia as subáreas onde ocorreram os maiores volumes de publicações nos últimos anos. O objetivo é ilustrar a concentração de pesquisas em temas, enquanto outros temas acabam tendo um volume de pesquisa muito pequeno. Observa-se que as subáreas finanças comportamentais e finanças ambientais não apresentaram nenhum artigo apresentado entre 2010 e 2012.

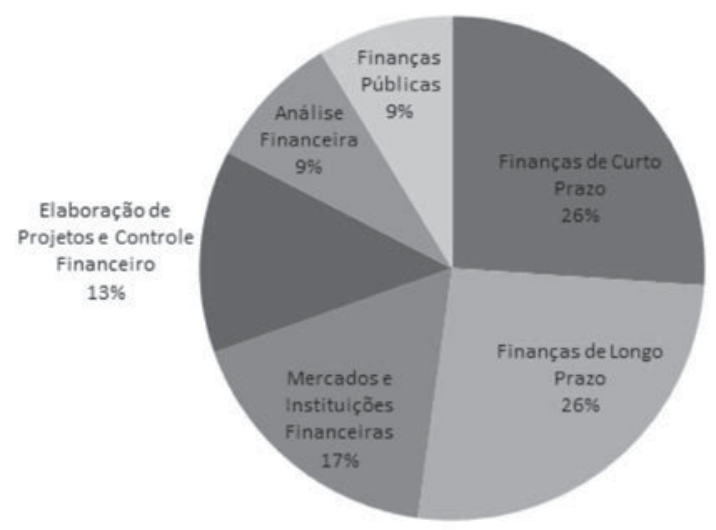

Gráfico 5: Concentração de temas nas subáreas Fonte: Elaborado pelos autores deste artigo

De 2010 até 2012 é possível observar uma concentração maior de pesquisas nas subáreas Finanças de Curto Prazo, Finanças de Longo Prazo e Mercados e Instituições Financeiras. 


\section{Considerações Finais}

O objetivo deste artigo consistia em evidenciar como estão as publicações em periódicos Qualis A no Brasil. Após o exposto na análise dos dados considera-se que o objetivo do artigo foi atingido.

Nos últimos anos, tanto a qualidade, quanto a quantidade das publicações cresceram significativamente. Novos temas passaram a fazer parte de objeto de pesquisa. A Região Sudeste detém a maior participação na busca de conhecimento na área. Alguns autores como Guimarães Jr. et al. (2006) implicam em alguns fatores que possam contribuir para esse perfil, como a Região Sudeste sediar a principal Bolsa de Valores do Brasil e a maior parte dos escritórios das principais Instituições Financeiras. Outros fatores seriam a preferência e a recorrência de temas pragmáticos, voltados para o mercado, presentes nas pesquisas.

Como principais achados do artigo destacam-se três. O primeiro é que entre 2010 até 2012 existe uma concentração maior de pesquisas nas subáreas Finanças de Curto Prazo, Finanças de Longo Prazo e Mercados e Instituições Financeiras. O segundo corresponde às instituições mais profícuas, que são a Universidade Presbiteriana Mackenzie e a Fundação Getúlio Vargas (EAESP/FGV). Em terceiro, as Revistas RAUSP e RAE apresentam a maior quantidade de artigos publicados sobre finanças.

Com a discriminação dos autores em gênero, verifica-se que os autores do sexo masculino publicaram, em média, 3,45 vezes mais do que os autores do sexo feminino. Porém cabe ressaltar que a quantidade de pesquisas em Finanças desenvolvidas por mulheres vem crescendo.

De forma geral, é possível afirmar que pesquisadores em finanças no Brasil apresentam tendência de serem do sexo masculino, pertencerem a uma instituição de ensino superior da Região Sudeste Sul e pesquisar os temas: Finanças de Curto Prazo, Finanças de Longo Prazo e Mercados e Instituições Financeiras.

\section{REFERÊNCIAS}

ASSAF NETO, Alexandre. Finanças corporativas e valor. 2. ed. São Paulo: Atlas, 2005.
BOTELHO, Louise Lira Roedel; CUNHA, Cristiano Castro de Almeida; MACEDO, Marcelo. O Método da revisão integrativa nos estudos organizacionais. Gestão e Sociedade, Belo Horizonte, v. 5, n. 11, p. 121-136, maio/ago., 2011.

BREALEY, R. A.; MYERS, S.C.; ALLEN, F. Principles of corporate finance. 8. ed., New York: McGraw-Hill, 2006.

FRANCO. Finanças ambientais: uma contribuição ao modelo de avaliação de risco de crédito agregando a variável ambiental. Dissertação, USP, 2009

GALBRAITH, J. K. Moeda: de onde veio, para onde foi. Tradução de Antônio Zoratto Sanvicente. São Paulo: Pioneira, 1977.

\section{GITMAN, L. J. Princípios de administração financeira.} 12. ed. São Paulo: Pearson, 2010.

GUIMARÃES Jr., Francisco Roberto F; CÂMARA, Samuel Façanha; PINHEIRO, Karine Bessa Porto. Perfil do pesquisador em finanças no Brasil. Gestão \& Regionalidade, São Caetano do Sul, RS, n. 63, jan.-abr. 2006.

HALFELD Mauro; TORRES, Fábio de Freitas Leitão.

Finanças comportamentais: aplicações no contexto brasileiro. Revista de Administração de Empresas, São Paulo, abr.-jun. 2001.

HERLING, L. H. D.; MORITZ, G. O.; LIMA; M.

V. Finanças corporativas: sua organização e base epistemológica. In: III COLÓQUIO INTERNACIONAL DE EPISTEMOLOGIA, Florianópolis, 2013. Anais... Florianópolis, 2013.

HUNT, Pearson. Financial policy of corporations.

Quarterly Journal of Economics, USA, LVII, p. 303313, Feb. 1943.

KEYNES. A teoria geral do emprego, juro e da moeda. 2. ed. São Paulo: nova cultural, 1985.

LEAL, Ricardo Pereira Câmara; SAITO, Richard. Finanças corporativas no Brasil. Revista de Administração de

Empresas Eletrônica, on-line, v. 2, n. 2, jul-dez., 2003.

LEMES Jr., A. B.; CHEROBIM, A. P.; RIGO, C. M.

Administração financeira: princípios, fundamentos $e$ práticas brasileiras. Rio de Janeiro: Campus, 2002. 
LUIZ, Alfredo José Barreto. Meta-análise: definição, aplicações e sinergia com dados espaciais. Cadernos de Ciência \& Tecnologia, Brasília, DF, v. 19, n. 3, p. 407428, set.-dez. 2002.

\section{MATIAS, A. B. (Org.). Finanças corporativas de curto} prazo. São Paulo: Atlas, 2006.

PIRES Valdemir. Finanças pessoais: Fundamentos e Dicas. Piracicaba SP, 2007. Disponível em: <http://www. pires.pro.br/documentos/livrofinpess67908.pdf $>$. Acesso em: 18 nov. 2012.

\section{ROBERTS, Richard. Por dentro das finanças}

internacionais: guia práticodos mercados e instituições financeiras. Rio de Janeiro: Jorge Zahar, 2000.

ROCHA, Irani et al. A presença da entropia da informação no controle orçamentário em ambiente inovador. Revista de Administração e Inovação, São Paulo, v. 8, n. 2, p.81-105, abr.-jun. 2011.

ROSS, Stephen A.; WESTERFIELD, Randolph W.; JAFFE F. Administração financeira. São Paulo: Atlas, 1995.

SANTOS, ALVES. O impacto da Lei de Responsabilidade Fiscal no desempenho financeiro e na execução orçamentária dos municípios no Rio Grande do Sul de 1997 a 2004. Revista de Administração Pública, Rio de Janeiro, v. 45, n.1, jan./fev. 2011.

SOLOMON, Ezra. Teoria da administração financeira. 3. ed. São Paulo: Zahar, 1977.

WESTON, J. Fred. Finanças de empresas: campo em metodologia. São Paulo: Atlas, 1977.

\section{Anexo A - Autores com MAIOR PRODUÇÃo}

\begin{tabular}{|c|c|c|}
\hline Autor & INSTITUIÇÃo & Número de Artigos \\
\hline Wesley Mendes-da-Silva & Universidade Presbiteriana Mackenzie & 4 \\
\hline Herbert Kimura & Universidade Presbiteriana Mackenzie & 3 \\
\hline Luiz Artur Ledur Brito & Fundação Getúlio Vargas - EAESP/FGV & 3 \\
\hline Wilson Toshiro Nakamura & Universidade Presbiteriana Mackenzie & 3 \\
\hline Adalberto Schnorrenberger & Univates & 2 \\
\hline Jairo Laser Procianoy & Universidade Federal do Rio Grande do Sul, & 2 \\
\hline John Robison & Unisersity of Texas & 2 \\
\hline Raimundo Christians & Prince Waterhouse & 2 \\
\hline Ricardo Pereira Câmara Leal & UFRJ Universidade Federal do Rio de Janeiro & 2 \\
\hline Saul B. Suslick & Universidade Estadual de Campinas/UNICAMP & 2 \\
\hline Stephen Limberg & Unisersityof Texas & 2 \\
\hline Adilson Soares & Secretaria Estadual da Saúde de São Paulo, & 1 \\
\hline Adriana Bruscato Bortoluzzo & Insper Instituto de Ensino e Pesquisa & 1 \\
\hline Alexandre Lemgruber Portugal d'Oliveira & ANVISA & 1 \\
\hline Ana Cecília Faveret de Sá Campelo & ANS & 1 \\
\hline Ana Luiza d'Ávila Viana & Universidade de São Paulo (USP) & 1 \\
\hline Ana Tereza da Silva Pereira & Fundação Cesgranrio & 1 \\
\hline
\end{tabular}




\begin{tabular}{|c|c|c|}
\hline Andre Carvalhal & PUC Rio Pontifícia Universidade Católica do Rio de Janeiro & 1 \\
\hline André Barreira da Silva Rocha & Universidade de Essex & 1 \\
\hline André Luís de Castro Moura Duarte & Insper Instituto de Ensino e Pesquisa & 1 \\
\hline AndreLuis Ferreira-Da-Silva & Universidade Federal do Rio Grande do Sul, & 1 \\
\hline Andrea Maria Accioly Fonseca Minardi & IBMEC SP & 1 \\
\hline Andressa Iovine Martins & Pontifícia Universidade Católica SP & 1 \\
\hline Anete Alberton & Universidade Federal de Santa Catarian - UFSC & 1 \\
\hline Antônio Carlos de Azevedo Sodré & Fundação Getúlio Vargas - EAESP/FGV & 1 \\
\hline Antônio de Pádua Alvarenga & Empresa Pesquisa Agropecuária de Minas Gerais - EPAMIG & 1 \\
\hline Ariovaldo dos Santos & Universidade de São Paulo/USP & 1 \\
\hline Artur Roberto do Nascimento & Universidade Estadual de Feira de Santana - UEFS. & 1 \\
\hline Augusto de Oliveira Monteiro & UNIFACS & 1 \\
\hline Aureliano Angel Bressan & Universidade Federal de Minas Gerais - UFMG & 1 \\
\hline Bettina Hedden-Dunkhorst & Federal Agency for Nature Conservation, Bonn, Germany & 1 \\
\hline Calebe da Costa Ferreira & Universidade do Grande Rio (Unigranrio) & 1 \\
\hline Camilo L. T. Andrade & Embrapa & 1 \\
\hline Carisi Anne Polanczyk & Universidade Federal do Rio Grande do Sul, & 1 \\
\hline Celma de Oliveira Ribeirob & Universidade de São Paulo/USP & 1 \\
\hline Celso da Costa Carrer & Universidade de São Paulo/USP & 1 \\
\hline César Augusto Tibúrcio Silva & UnB Universidade de Brasília & 1 \\
\hline Chen Yen-Tsang & Fundação Getúlio Vargas - EAESP/FGV & 1 \\
\hline Claudinê Jordão de Carvalho & Universidade Federal de Uberlândia - UFU & 1 \\
\hline Claudio Henrique da Silveira Barbedo & Instituto Brasileiro de Mercado de Capitais - IBMEC-RJ & 1 \\
\hline Cristiano Augusto Borges Forti & Fundação Getúlio Vargas - EAESP/FGV & 1 \\
\hline Daniel Carrasqueira de Moraes & Universidade Presbiteriana Mackenzie & 1 \\
\hline Daniel Reed Bergmann & Fundação Instituto de Administração (FIA) & 1 \\
\hline Dante Mendes Aldrighi & Universidade de São Paulo/USP & 1 \\
\hline Déa Mara Tarbes de Carvalho & Conselho Nacional de Secretários de Saúde & 1 \\
\hline Delane Botelho & Fundação Getúlio Vargas - EAESP/FGV & 1 \\
\hline Diego Paraiso Garcia Guimarães & $\begin{array}{l}\text { Associação Nacional das Instituições do } \\
\text { Mercado Financeiro - ANDIMA }\end{array}$ & 1 \\
\hline Dinorá Elite Floriani & Univali & 1 \\
\hline Edson Luis Kammler & $\mathrm{UnC}$ & 1 \\
\hline
\end{tabular}




\begin{tabular}{|c|c|c|}
\hline Eduardo P. Peres & IBMEC RJ & 1 \\
\hline Eduardo Senra Coutinho & Universidade Federal de Minas Gerais - UFMG & 1 \\
\hline Elizabeth Krauter & Universidade de São Paulo/USP & 1 \\
\hline Elmo Tambosi Filho & Universidade Federal de Santa Catarian - UFSC & 1 \\
\hline Élvia Fadul & UNIFACS & 1 \\
\hline Emanuel Junqueira & Universidade de São Paulo/USP & 1 \\
\hline Étienne Henry & Fundação Getúlio Vargas - EAESP/FGV & 1 \\
\hline Euler Albergaria de Melo & UNIFACS & 1 \\
\hline Fábio de Freitas Leitão Torres & Consultor da Booz-Allen \& Hamilton & 1 \\
\hline Fábio Frezatti & Universidade de São Paulo/USP & 1 \\
\hline Fábio Gallo Garcia & Fundação Getúlio Vargas - EAESP/FGV & 1 \\
\hline Fabio R. Chaddad & Universityof Missouri - Columbia & 1 \\
\hline Fátima Scarparo Cunha & Universidade Federal do Rio de Janeiro & 1 \\
\hline Felipe Faissol Janot de Matos & Universidade Federal do Rio de Janeiro - UFRJ & 1 \\
\hline Fernanda Fernandes Rodrigues & UnB Universidade de Brasília & 1 \\
\hline Fernanda Finotti Cordeiro Perobelli & Fundação Getúlio Vargas - EAESP/FGV & 1 \\
\hline Fernanda Maciel Peixoto & Universidade Federal de Minas Gerais - UFMG & 1 \\
\hline Fernando Coelho Martins Ferreira & Fundação Getúlio Vargas - EAESP/FGV & 1 \\
\hline Flávia Tavares Silva Elias & Ministério da Saúde & 1 \\
\hline Flávio Carvalho de Vasconcelos & Fundação Getúlio Vargas - EAESP/FGV & 1 \\
\hline Francisco Marcelo Barone & Universidade Federal Fluminense - UFF & 1 \\
\hline Francisco Nepomuceno Filho & Petrobras & 1 \\
\hline Gabriel Alves da Costa Lima & Universidade Estadual de Campinas/UNICAMP & 1 \\
\hline Gabriela Aferri & Universidade de São Paulo/USP & 1 \\
\hline George Edward Machado Kornis & Universidade do Estado do Rio de Janeiro, & 1 \\
\hline Germano Mendes de Paula & Universidade Federal de Uberlândia - UFU & 1 \\
\hline Glauber dos Santos & Universidade Federal de Lavras - UFLA & 1 \\
\hline Guilherme Beil Amado & Universidade Federal de Lavras - UFLA & 1 \\
\hline Guilherme Silveira Martins & Insper Instituto de Ensino e Pesquisa & 1 \\
\hline Gustavo Silva Araújo & Instituto Brasileiro de Mercado de Capitais - IBMEC-RJ & 1 \\
\hline Gustavo Zoio Portela & Fundação Oswaldo Cruz & 1 \\
\hline Haroldo de Paiva Nogueira & Universidade Federal de Viçosa - UFV & 1 \\
\hline
\end{tabular}


Luiz Henrique Debei Herling • Gilberto de Oliveira Moritz • Thiago Coelho Soares • Marcus Venícius Andrade de Lima

\begin{tabular}{|c|c|c|}
\hline Hélio de Paula Leite & Fundação Getúlio Vargas - EAESP/FGV & 1 \\
\hline Hudson Fernandes Amaral & Universidade Federal de Minas Gerais - UFMG & 1 \\
\hline Ivan Pinto Dias & Fundação Getúlio Vargas - EAESP/FGV & 1 \\
\hline Janann Joslin Medeiros & UnB Universidade de Brasília & 1 \\
\hline João C. F. Borges Júnior & Universidade Federal de Pernambuco - UFPE & 1 \\
\hline João Carlos Hopp & Fundação Getúlio Vargas - EAESP/FGV & 1 \\
\hline João Henrique Gurtler Scatena & Universidade Federal de Mato Grosso, & 1 \\
\hline João Luiz Cardoso & Universidade Estadual de Campinas/UNICAMP & 1 \\
\hline João Mario Csillag & Fundação Getúlio Vargas - EAESP/FGV & 1 \\
\hline Jomar Miranda Rodrigues & UnB Universidade de Brasília & 1 \\
\hline José Alonso Borba & Universidade Federal de Santa Catarian - UFSC & 1 \\
\hline José Alves Dantas & Centro Universitário Unieuro & 1 \\
\hline José Augusto Veiga da Costa Marques & Universidade Federal do Rio de Janeiro - UFRJ & 1 \\
\hline José Lúcio Tozetti Fernandes & UnB Universidade de Brasília & 1 \\
\hline José Mendes Ribeiro & Fundação Oswaldo Cruz & 1 \\
\hline José Noronha & Fundação Oswaldo Cruz & 1 \\
\hline José Paulo Alves Fusco & Universidade de São Paulo/USP & 1 \\
\hline José Rivaldo Melo de França & Ministério da Saúde. & 1 \\
\hline José Roberto Ferreira Savoia & Universidade de São Paulo (USP) & 1 \\
\hline José Roberto Securato & Universidade de São Paulo/USP & 1 \\
\hline Joshua Onome Imoniana & Universidade Presbiteriana Mackenzie & 1 \\
\hline Juliana Bonomi Santos & Lancaster University Management School - UK & 1 \\
\hline Laercio Antônio Gonçalves Jacovine & Universidade Federal de Viçosa, UFV & 1 \\
\hline Leonardo Augusto Soares Ferreira & Universidade de São Paulo (USP) & 1 \\
\hline Leonardo Fernando Cruz Basso & Universidade Presbiteriana Mackenzie & 1 \\
\hline Lenos Trigeorgis & UniversityofCyprus & 1 \\
\hline Ligia Maura Costa & Fundação Getúlio Vargas - EAESP/FGV & 1 \\
\hline Lucia Barros & Fundação Getúlio Vargas - EAESP/FGV & 1 \\
\hline Lucio Rodrigues Capelletto & Universidade de Brasília - UnB & 1 \\
\hline Lucy Aparecida de Sousa & Fundação Armando Álvares Penteado & 1 \\
\hline Luís Fernando Moreira & UNIOESTE e UNIPAR & 1 \\
\hline Luis Gustavo Do Lago Quinteiro & UnB Universidade de Brasília & 1 \\
\hline
\end{tabular}




\begin{tabular}{|c|c|c|}
\hline Luiz Carlos Di Serio & Insper Instituto de Ensino e Pesquisa & 1 \\
\hline Luiz Claudio de Souza Campos & Instituto Brasileiro de Mercado de Capitais - IBMEC-RJ & 1 \\
\hline Luiz Flavio Autran Monteiro Gomes & Instituto Brasileiro de Mercado de Capitais - IBMEC-RJ & 1 \\
\hline Luiz Maurício Franco Moreira & Banco Central & 1 \\
\hline Luiz Roberto Salgado Candiota & Consultor da Booz-Allen \& Hamilton & 1 \\
\hline Manuel José da Rocha Armada & Universidade de Coimbra & 1 \\
\hline Marcelo Alvaro da Silva Macedo & Universidade Federal do Rio de Janeiro - UFRJ & 1 \\
\hline Marcelo Machado de Luca Oliveira Ribeiro & Universidade de São Paulo/USP & 1 \\
\hline Márcio Lopes da Silva & Universidade Federal de Viçosa - UFV & 1 \\
\hline Marcos Antônio de Camargos & Universidade Federal de Minas Gerais - UFMG & 1 \\
\hline Marcos Aurélio Lopes & Universidade Federal de Lavras - UFLA & 1 \\
\hline Maria de Fátima Siliansky de Andreazzi & Universidade Federal do Rio de Janeiro & 1 \\
\hline Maria Tereza Fleury & Universidade de São Paulo/USP & 1 \\
\hline Maryse Farhi & Universidade Estadual de Campinas/UNICAMP & 1 \\
\hline Mauri Aparecido de Oliveira & Universidade Federal de São Paulo (UNIFESP) & 1 \\
\hline Mauro Halfeld & Universidade Federal do Paraná - UFPR & 1 \\
\hline Moacir Sancovschi & Universidade Federal do Rio de Janeiro & 1 \\
\hline Murillo Valverde Lima & Fundação Getúlio Vargas - EAESP/FGV & 1 \\
\hline Nadia Jorge Diniz de Oliveira & Universidade de São Paulo/USP & 1 \\
\hline Nathalie Vicente Nakamura Palombini & Universidade Presbiteriana Mackenzie & 1 \\
\hline Newton Carneiro Afonso da Costa Jr & Universidade Federal de Santa Catarian - UFSC & 1 \\
\hline Nilson do Rosário Costa & Fundação Oswaldo Cruz & 1 \\
\hline Oswaldo Yoshimi Tanaka & Universidade de São Paulo (USP) & 1 \\
\hline Otavio Ribeiro De Medeiros & UnB Universidade de Brasília & 1 \\
\hline Paulo A. Ferreira & Universidade Federal de Viçosa - UFV & 1 \\
\hline Paulo Azevedo de Oliveira Magalhães Filho & FIR & 1 \\
\hline Rafael Bisinha & Banco Itaú & 1 \\
\hline Rafael Felipe Schiozer & Fundação Getúlio Vargas (EAESP-FGV) & 1 \\
\hline Rafael G. Burstein Goldszmidt & Fundação Getúlio Vargas - EBAPE/FGV & 1 \\
\hline Renato Aparecido Aguiar & Universidade de São Paulo/USP & 1 \\
\hline Ricardo D. Brito & IBMEC SP & 1 \\
\hline Richard Saito & Fundação Getúlio Vargas - EAESP/FGV & 1 \\
\hline Robert Aldo Iquiapaza & Universidade Federal de Minas Gerais - UFMG & 1 \\
\hline
\end{tabular}


Luiz Henrique Debei Herling • Gilberto de Oliveira Moritz • Thiago Coelho Soares • Marcus Venícius Andrade de Lima

\begin{tabular}{|c|c|c|}
\hline Roberto Alexandre Zanchetta Borghi & Universidade Estadual de Campinas/UNICAMP & 1 \\
\hline Roberto Braga & Universidade de São Paulo/USP & 1 \\
\hline Roberto Moura Sales & Universidade de São Paulo/USP & 1 \\
\hline Rodrigo Antonini Ribeiro & Universidade Federal do Rio Grande do Sul, & 1 \\
\hline Rodrigo Dill & Universidade Federal de Santa Catariana - UFSC & 1 \\
\hline Rondineli Mendes da Silva & Universidade do Estado do Rio de Janeiro, & 1 \\
\hline Rosangela Caetano & Universidade do Estado do Rio de Janeiro & 1 \\
\hline Rosemarie Bröker Bone & Universidade Federal do Rio de Janeiro - UFRJ & 1 \\
\hline Rubens Famá & Universidade de São Paulo/USP & 1 \\
\hline Sebastião Renato Valverde & Universidade Federal de Viçosa - UFV & 1 \\
\hline Sergio Del Porto Santos & Fundação Getúlio Vargas - EAESP/FGV & 1 \\
\hline Sérgio Giovanetti Lazzarini & Washington University & 1 \\
\hline Sérgio Jurandyr Machado & Insper Instituto de Ensino e Pesquisa & 1 \\
\hline Sigismundo Bialoskorski Neto & Universidade de São Paulo (USP) & 1 \\
\hline Silvia Quiota & Fundação Getúlio Vargas - EAESP/FGV & 1 \\
\hline Sulamis Dain & Universidade do Estado do Rio de Janeiro, & 1 \\
\hline Susana Jorge & Universidade de Coimbra & 1 \\
\hline Tânia Regina Sordi Relvas & Universidade de São Paulo/USP & 1 \\
\hline Telma Ruth Pereira & Fundação Cesgranrio & 1 \\
\hline Thalita Amorim Vaz & Insper Instituto de Ensino e Pesquisa & 1 \\
\hline Tiago Wickstrom Alves & Universidade do Vale do Rio dos Sinos & 1 \\
\hline Vânia Cristina Canuto Santos & Ministério da Saúde & 1 \\
\hline Verônica Ines Fernandez Orellano & Fundação Getúlio Vargas - EAESP/FGV & 1 \\
\hline Wellington Pinto & $\begin{array}{l}\text { Faculdade do Meio-Ambiente e Tecnologia } \\
\text { de Negócios - FAMATEC }\end{array}$ & 1 \\
\hline Willian Eld Junior & Fundação Getúlio Vargas - EAESP/FGV & 1 \\
\hline
\end{tabular}

